\title{
ON HUA'S INEQUALITY IN REAL INNER PRODUCT SPACES
}

\author{
S. S. DRAGOMIR AND G.-S. YANG
}

\begin{abstract}
A generalization of the celebrated Hua's inequality in real inner product spaces and some connected results are given.
\end{abstract}

\section{Introduction}

The following inequality due to Lo-Keng Hua is important in Number Theory [1]:

$$
\left(\delta-\sum_{i=1}^{n} x_{i}\right)^{2}+\alpha \sum_{i=1}^{n} x_{i}^{2} \geq k_{n} \delta^{2}
$$

where $\delta, \alpha>0, x_{i} \in \mathbb{R}(i=1,2, \ldots, n)$ and $k_{n}:=\alpha(n+\alpha)^{-1}$ with equality if and only if $x_{i}=h_{n} \delta$ where $h_{n}=(n+\alpha)^{-1}$.

Recently, Chung-Lie Wang [2] has given the following interesting generalization of (1.1):

Theorem 1.1. Let $\delta, \alpha$ be as above, Then for $p>1$, the inequality

$$
\left(\delta-\sum_{i=1}^{n} x_{i}\right)^{p}+\alpha^{p-1} \sum_{i=1}^{n} x_{i}^{p} \geq k_{n}^{p-1} \delta^{p}
$$

holds, for all nonnegative $x_{i} \in \mathbb{R}(i=1,2, \ldots, n)$ with $\sum_{i=1}^{n} x_{i} \leq \delta$. The sign of inequality in (1.2) is reversed for $0<p<1$. In either case, the sign of equality holds in (1.2) iff $x_{i}=h_{n} \delta(i=1,2, \ldots, n)$.

An integral variant is also given.

The main aim of this paper is to extend (1.1) in real inner product linear spaces. Some inequalities in connection with Hua's result are also given.

Received December 27, 1994

1991 Mathematics Subject Classification. Primary 26D15, 46C20

Key words and phrases. Hua's inequality, Schwarz's inequality, Inner product spaces. 


\section{The Main Results}

We will start with the following theorem containting a consistent generalization of (1.1):

Theorem 2.1 Let $(X ;()$,$) be a real inner product space and \alpha>0, \delta>0$. For all $x, y \in X$ one has the inequality:

$$
(\delta-(x, y))^{2}+\alpha\|x\|^{2} \geq \frac{\alpha \delta^{2}}{\alpha+\|y\|^{2}} .
$$

The sign of equality holds in (2.1) iff

$$
x=\frac{\delta}{\alpha+\|y\|^{2}} y .
$$

Proof. If $y=0$, the inequaltiy (2.1) is obvious.

Suppose that $y \neq 0$. Then by the well-known Schwarz's inequality in inner product spaces have:

$$
(\delta-(x, y))^{2}+\alpha\|x\|^{2} \geq(\delta-(x, y))^{2}+\frac{\alpha}{\|y\|^{2}}(x, y)^{2}
$$

with equality iff there exists a real number $\lambda$ so that $x=\lambda y$. state:

Denote $z=(x, y), \alpha^{\prime}=\frac{\alpha}{\|y\|^{2}}$. Applying Lo-Keng Hua's inequality for $n=1$, we can

$$
(\delta-z)^{2}+\alpha^{\prime} z^{2} \geq \frac{\alpha^{\prime}}{1+\alpha^{\prime}} \delta^{2}
$$

with equlaity iff

$$
z=\frac{\delta}{1+\alpha^{\prime}}
$$

Hence

$$
(\delta-(x, y))^{2}+\frac{\alpha}{\|y\|^{2}}(x, y)^{2} \geq \frac{\alpha}{\alpha+\|y\|^{2}} \delta^{2}
$$

with equlity iff

$$
(x, y)=\frac{\delta\|y\|^{2}}{\alpha+\|y\|^{2}} .
$$

Since $x=\lambda y$, then by the above condition we deduce that

$$
\lambda\|y\|^{2}=\frac{\delta\|y\|^{2}}{\alpha+\|y\|^{2}}
$$

which gives us $\lambda=\frac{\delta}{\alpha+\|y\|^{2}}$, and the theorem is thus proved. 
Remark 2.2. If we assume that $X \in \mathbb{R}^{n},($,$) is the usual inner product in \mathbb{R}^{n}$, i.e., $(x, z)=\sum_{i=1}^{n} x_{i} z_{i}$ for $x=\left(x_{1}, \ldots, x_{n}\right), z=\left(z_{1}, \ldots, z_{n}\right) \in R^{n}$ and $y=(1, \ldots, 1)$, then by Theorem 2.1 we recapture Lo-Keng Hua's result (1.1).

Corollary 2.3. Let $(X,()$,$) be an inner product space and \delta, \alpha>0$. Then one has the inequality:

$$
\left(\delta-\sum_{i=1}^{n}\left(x_{i}, y_{i}\right)\right)^{2}+\alpha \sum_{i=1}^{n}\left\|x_{i}\right\|^{2} \geq \frac{\alpha \delta^{2}}{\alpha+\sum_{i=1}^{n}\left\|y_{i}\right\|^{2}}
$$

for all $x_{i}, y_{i} \in X(i=1,2, \ldots, n)$. The case of equality holds in (2.2) iff

$$
x_{i}=\frac{\delta}{\alpha+\sum_{i=1}^{n}\left\|y_{1}\right\|^{2}} y_{i} \quad \text { for all } i \in\{1, \ldots, n\} .
$$

Proof. Consider the linear space $X^{n}:\left\{\left(x_{1}, \ldots, x_{n}\right) \mid x_{i} \in X\right\}$. Then the mapping $[]:, X^{n} \times X^{n} \rightarrow \mathbb{R}$ given by:

$$
[Z, Y]:=\sum_{i=1}^{n}\left(z_{i}, y_{i}\right), \quad Z=\left(z_{1}, \ldots, z_{n}\right), \quad Y=\left(y_{1}, \ldots, y_{n}\right)
$$

is an inner product on $X^{n}$. Applying the inequality (2.1) for the inner product $[$,$] we$ obtain the desired inequality.

The following lemma is interesting in itself.

Lemma 2.4. Let $(X ;()$,$) be a real inner product space and \alpha>0$. Then for all $x, y \in X$ we have the inequality:

$$
\|y-x\|^{2}+\alpha\|x\|^{2} \geq \frac{\alpha}{1+\alpha}\|y\|^{2}
$$

with equality iff

$$
x=\frac{1}{1+\alpha} y .
$$

Proof. Firstly, we observe that in every normed linear space we have the inequaltiy:

$$
\|y-x\|^{2} \geq(\|y\|-\|x\|)^{2} \quad \text { for all } x, y \in X .
$$

Since $(X ;())$ is assumed to be an inner product space, hence the equality holds in $(2.4)$ iff

$$
(x, y)=\|x\|\|y\|,
$$

i.e., there exists a real number $\lambda \geq 0$ so that $x=\lambda y$. 
Now, by Hua's inequality for $n=1$, we can write:

$$
\|y-x\|^{2}+\alpha\|x\|^{2} \geq(\|y\|-\|x\|)^{2}+\alpha\|x\|^{2} \geq \frac{\alpha}{1+\alpha}\|y\|^{2}
$$

with equality in the second inequality iff

$$
\|x\|=\frac{1}{1+\alpha}\|y\|
$$

Since $x=\lambda y$, we deduce by the above equality that $\lambda=\frac{1}{1+\alpha}$ and the lemma is thus proved.

Remark 2.5. The inequality (2.3) holds in every normed linear space, but the case of equality does not remain true in the general situation. For example, let $X=C[0,1]$, with $\|f\|=\int_{0}^{1}|f(t)| d t$, if $f \in X$. Let $f$ be any positive function in $X$ and $g=f+1$. Then $\|f-g\|^{2}=(\|f\|-\|g\|)^{2}=1$, but $f \neq \lambda g$ for any real $\lambda$.

Remark 2.6. If we assume that $X=\mathbb{R}^{n}, y=\left(\delta_{1}, \ldots, \delta_{n}\right)$ then by the above lemma we obtain the following inequality of Hua's type:

$$
\sum_{i=1}^{n}\left(\delta_{i}-x_{i}\right)^{2}+\alpha \sum_{i=1}^{n} x_{i}^{2} \geq \frac{\alpha}{1+\alpha} \sum_{i=1}^{n} \delta_{i}^{2}
$$

with equality iff

$$
x_{i}=\frac{\delta_{i}}{1+\alpha}, \quad i=1,2, \ldots, n .
$$

Now, provided that $\delta_{1}=\cdots=\delta_{n}=\delta$, then

$$
\sum_{i=1}^{n}\left(\delta-x_{i}\right)^{2}+\alpha \sum_{i=1}^{n} x_{i}^{2} \geq \frac{n \alpha \delta^{2}}{1+\alpha}
$$

with equality iff

$$
x_{i}=\frac{\delta}{1+\alpha}, \quad i=1,2, \ldots, n .
$$

Finally, we also have the following generalization of Hua's inequaltiy:

Theorem 2.7. Let $(X ;()$,$) be an inner product space and x_{i} \in X(i=$ $1,2, \ldots, n), y \in X$ and $\alpha>0$. Then one has the inequality:

$$
\left\|y-\sum_{i=1}^{n} x_{i}\right\|^{2}+\alpha \sum_{i=1}^{n}\left\|x_{i}\right\|^{2} \geq \frac{\alpha}{n+\alpha}\|y\|^{2} .
$$

The equality holds in (2.4) iff

$$
x_{i}=\frac{1}{n+\alpha} y \quad \text { for all } i=1,2, \ldots, n .
$$


Proof. By the well-known Cauchy-Buniakowski-Schwarz's inequality for real numbers, we have:

$$
n \sum_{i=1}^{n} a_{i}^{2} \geq\left(\sum_{i=1}^{n} a_{i}\right)^{2}
$$

with equality iff $a_{i}=k, k \in \mathbb{R}$ for all $i=1, \ldots, n$; we have that

$$
n \sum_{i=1}^{n}\left\|x_{i}\right\|^{2} \geq\left(\sum_{i=1}^{n}\left\|x_{i}\right\|\right)^{2}
$$

with equality iff $\left\|x_{i}\right\|=\lambda \neq 0$ for all $i=1,2, \ldots, n$.

Without loss the generality, we can assume in what follows that $x_{i} \neq 0$ for all $i=1,2, \ldots, n$. Thus we have:

$$
\left\|y-\sum_{i=1}^{n} x_{i}\right\|^{2}+\alpha \sum_{i=1}^{n}\left\|x_{i}\right\|^{2} \geq\left\|y-\sum_{i=1}^{n} x_{i}\right\|^{2}+\frac{\alpha}{n}\left(\sum_{i=1}^{n}\left\|x_{i}\right\|\right)^{2}
$$

with equality iff $\left\|x_{i}\right\|=\lambda>0$ for all $i \in\{1, \ldots, n\}$.

By the generalized triangle inequality we have:

$$
\left\|y-\sum_{i=1}^{n} x_{i}\right\|^{2}+\frac{\alpha}{n}\left(\sum_{i=1}^{n}\left\|x_{i}\right\|\right)^{2} \geq\left\|y-\sum_{i=1}^{n} x_{i}\right\|^{2}+\frac{\alpha}{n}\left\|\sum_{i=1}^{n} x_{i}\right\|^{2}
$$

with equality iff

$$
\left\|\sum_{i=1}^{n} x_{i}\right\|=\sum_{i=1}^{n}\left\|x_{i}\right\|
$$

Since $X$ is assumed to be an inner product space, then the above equaltiy holds iff

$$
0=\left(\sum_{i=1}^{n}\left\|x_{i}\right\|\right)^{2}-\left\|\sum_{i=1}^{n} x_{i}\right\|^{2}=\sum_{\substack{i, j=1 \\ i \neq j}}^{n}\left(\left\|x_{i}\right\|\left\|x_{j}\right\|-\left(x_{i}, x_{j}\right)\right) .
$$

But, by Schwarz's inequality we have:

$$
\left\|x_{i}\right\|\left\|x_{j}\right\|-\left(x_{i}, x_{j}\right) \geq 0 \text { for all } i, j \in\{1, \ldots, n\}
$$

and thus (2.7) holds iff

$$
\left\|x_{i}\right\|\left\|x_{j}\right\|=\left(x_{i}, x_{j}\right) \text { for all } i, j \in\{1, \ldots, n\}, i \neq j
$$

i.e., there exists $\lambda_{i j}>0$ so that $x_{i}=\lambda_{i j} x_{j}$ for all $i, j \in\{1, \ldots, n\}$ with $i \neq j$. 
Consequently, by the inequalities (2.5) and (2.6) we have that:

$$
\left\|y-\sum_{i=1}^{n} x_{i}\right\|^{2}+\alpha \sum_{i=1}^{n}\left\|x_{i}\right\|^{2} \geq\left\|y-\sum_{i=1}^{n} x_{i}\right\|^{2}+\frac{\alpha}{n}\left\|\sum_{i=1}^{n} x_{i}\right\|^{2}
$$

with equality iff $\left\|x_{i}\right\|=\lambda>0$ and $x_{i}=\lambda_{i j} x_{j}$ for all $i, j \in\{1, \ldots, n\}$ with $i \neq j$ and $\lambda_{i j}>0$. Thus

$$
\lambda=\left\|x_{i}\right\|=\lambda_{i j}\left\|x_{j}\right\|=\lambda_{i j} \lambda \text { for } i, j \subseteq\{1, \ldots, n\}, i \neq j
$$

which gives us $\lambda_{i j}=1$ for $i, j \in\{1, \ldots, n\}(i \neq j)$ and then $x_{i}=x \in X$ for all $i \in$ $\{1, \ldots, n\}$.

Now, using Lemma 2.4, we have that:

$$
\left\|y-\sum_{i=1}^{n} x_{i}\right\|^{2}+\frac{\alpha}{n}\left\|\sum_{i=1}^{n} x_{i}\right\|^{2} \geq \frac{\alpha}{n+\alpha}\|y\|^{2}
$$

with equality iff

$$
\sum_{i=1}^{n} x_{i}=\frac{n}{n+\alpha} y
$$

In conclusion, by the inequalities (2.8) and (2.9) we have that

$$
\left\|y-\sum_{i=1}^{n} x_{i}\right\|^{2}+\alpha \sum_{i=1}^{n}\left\|x_{i}\right\|^{2} \geq \frac{\alpha}{n+\alpha}\|y\|^{2},
$$

with equality iff $x_{i}=x(i \in\{1, \ldots, n\})$ and $\sum_{i=1}^{n} x_{i}=\frac{n}{n+\alpha} y$. i.e.,

$$
x_{i}=x=\frac{1}{n+\alpha} y \quad \text { for all } i \in\{1, \ldots, n\},
$$

and the theorem is proved.

Remark 2.8. If $X \in \mathbb{R}, y=\delta>0$ and $(x, z):=x z, x, z \in \mathbb{R}$. then by the above theorem we recapture Hua's inequality (1.1) with the same case of equality.

\section{References}

[1] L. K. Hua, Additive Theory of Prime Numbers (Translated by N. B. Ng) in Translations of Math. Monographs, Vol. 13, Amer. Math. Soc. Providence, RI, 1965.

[2] C.-L. Wang, "Lo-keng Hua inequality and dynamic programming," J. Math. Anal. Appl., 166(1992), 345-350.

Departmetn of Mathematics, Timisoara University, B-dul V. Parvan No.4, RO-1900 Tiimisoara, Romania.

Department of Mathematics, Tamkang University, Tamsui, Taiwan 25137, Republic of China. 